\title{
A nonlinear state feedback for DC/DC boost converters
}

\author{
O. Gehan, E. Pigeon, T. Menard; M. Pouliquen, \\ GREYC, University of Caen, \\ $6 \mathrm{Bd}$ du Maréchal Juin, \\ 14050 Caen Cedex, \\ France. \\ e-mail: tomas.menard@unicaen.fr \\ H. Gualous, Y. Slamani, B. Tala-Ighil, \\ LUSAC, University of Caen, Rue Louis Aragon \\ 50130 Cherbourg Octeville \\ France)
}

\begin{abstract}
This paper investigates the control problem for static boost type converters using an high gain state feedback robust controller incorporating an integral action. The robust feature allows to achieve the required performance in presence of parametric uncertainties, while the integral action provides an offset free performance with respect to the desired levels of voltage. The adopted high gain approach is motivated by both fundamental as well as practical considerations, namely the underlying fundamental potential and the design parameter specification simplicity. The stability and convergence analysis has been carried out using an adequate Lyapunov approach, and the control system calibration is achieved throughout a few design parameters which are closely related to the desired dynamical performances. The effectiveness of the proposed control approach has been corroborated by numerical simulations and probing experimental results.
\end{abstract}

\section{Introduction}

The control of static converters has been the subject of an important research activity over the past decades. This interest is mainly due to the emergence of embedded electronics in everyday life, increasing thereby the need for more efficient converters. Indeed, this type of converter is used for many applications such as laptop computers [1], photo-voltaic [2], vehicular systems [3], fuel cell [4], etc. Furthermore in recent use of electrical devices, the control problem is not the only feature required from the user. There are more and more need of informations on the evolution/aging of the system. In the case of converters, these informations can be obtained by reconstructing voltages and currents thanks to suitable observers.

Of particular interest is the Boost Converter which maintains a low power loss in the conversion process. The counterpart is that the average model presents strong nonlinearities due to the presence of bilinear terms and unstable zero dynamics which reduce the control system performances. These difficulties have given rise to several control solutions involving the converter output voltage. A widely spread method is the current-mode control which consists of two nested closed loop systems where the inner one is a current control system, while the outer one is a voltage control system ( [5-7]). The feasibility of the nested control system require the inner control to be relatively faster. Furthermore, it stabilizes the unstable zero dynamics, allowing thereby to apply usual control designs. However the control system performances may be poor when working out of the nominal operating conditions. Even so, this approach, commonly used in the industry, has been developed in many regards since then ( [8-11]).

\footnotetext{
${ }^{*}$ Corresponding author
} 
Several approaches have been recently proposed to circumvent the limitations of the usual methods from both performance improvement and simplicity points of views. They are based on direct output voltage control without resorting to the current control system as well as the stabilization of unstable zeros dynamics. A common technique consists in using the linearized model of the system, which is valid in a neighborhood of the nominal point, with its uncertainties if needed. Some models for these uncertainties have been proposed in [12]. LQR controllers have been used in [13,14] and [15] under small signals constraints for construction purposes. In order to cope with large signal disturbances, the linear techniques have been adapted in different ways that can be classified into two main streams. The first approach consists in designing a robust linear controller. Robust linear state feedback have been designed in [16] using passivity and using LMI in [17-20]. These approaches necessarily involve a trade-off between performances and robustness since the structure of the controller is linear. Different works have been done in order to keep the simplicity of the control law, while having better performances. Auto-tunning PID have been considered in [21,22]. The extended linearization with a PI has been pursued in [23] and the model predictive control has been considered in [24]. Although, these approaches increase the robustness and performances to large disturbances, they would exhibit poor performances on the whole working space and can lead to unexpected behavior.

Given the nonlinear structure of the average model of the boost converter, numerous approaches using nonlinear control law have been proposed in the literature. Early approaches such as passivity based control and Lyapunov based control have been considered in [25-27]. These methods have shown to be more robust to large signals disturbances but their performances cannot be compared to the standard techniques. More complicated techniques have then been used in order to achieve better performances using Hammerstein models ( $[28,29])$ or time optimal control $([30,31])$. It is however worth noticing that these techniques need a very precise knowledge of the model which is difficult since the model is usually an average model (this model does not take into account all the uncertainties on the components). Backstepping control has been used in [32] for current control, the voltage value is indirectly determined from the current using the model knowledge and henceforth the precision on the desired voltage directly relies on the model's accuracy. Fuzzy controllers have been used in [33-35] and [36] without any guarantee of stability and performance. Various contributions have been devoted to the sliding mode control approach for its robustness to bounded uncertainties [37-41]. They suffer from implementation issues because of the chattering behavior occurring near the sliding surface.

The objective of this paper is to propose a state feedback which is easy to calibrate, robust to uncertainties and competitive with respect to classical methods such as current mode control. Of fundamental interest, it performs well on the whole working space and is theoretically guaranteed. In order to cope with the unstable zeros dynamics, only regulation of the output voltage at a constant value is considered, as in most of the applications of this boost converter. The controller consists in combining an integral high-gain state feedback control law with an high-gain observer. Only three design parameters have to be specified and these parameters have physical meaning and are closely related to the underlying control dynamical performance. The admissible assumption of having access to on-line measurements of the current in the inductor and the output voltage is considered here. The current delivered to the load and the input voltage are not measured and are then estimated by a high-gain observer. In most approaches, the robustness toward changes in the current in the inductor and the output voltage is obtained through a degradation of the performances, since they are supposed to be unknown. This is not the case here, since these informations are reconstructed and used in the control law. It should be noted that these informations could be further use for fault detection purposes.

This paper is organized as follows. In section 2, the dynamical average model of the boost converter is presented. In the next section, an high gain type control law is synthesized, its construction is split into three parts. First the nominal case is considered and a control law is designed, then an observer reconstruct the current delivered to the load and the output voltage, finally, these two parts are mixed in order to obtain a robust state feedback. Section 4 is dedicated to experimental results. Finally, section 5 concludes the paper.

\section{Modeling of boost converter}

The electrical model of the Boost converter considered in this paper is shown on Fig. 1. It consists of an inductor $L$, a capacitor $C$ and a controlled switch. The output current is denoted $i(t)$ and the control variable $u(t)$ represents the duty cycle of the controlled IGBT. The first objective is to keep the converter's output voltage $v_{c}(t)$ at a constant reference value $v_{c}^{*}$, independently of the disturbances that could affect the operation of the converter, namely, the variations of the supply voltage of the battery and the load. This control objective is feasible, while tracking a reference sequence voltage is not possible in this form as the zero dynamics are not stable. For cost reasons and simplicity of implementation, the output voltage and the coil current are the unique available measurements. The second objective is to deliver an on line estimation of the unmeasured variables, namely the battery voltage and the output current for process monitoring considerations.

To achieve these objectives, the average model considered in this paper directly includes the losses and can thus be 


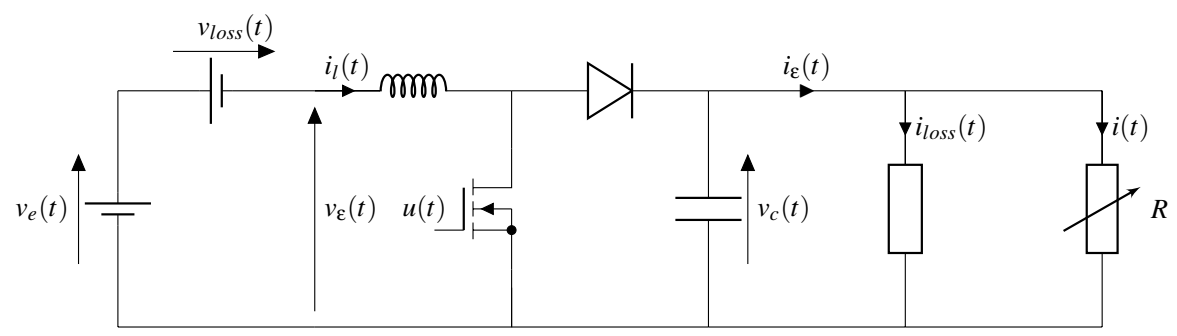

Fig. 1: Electric schematic of the Boost converter

written into the following form:

$$
\left\{\begin{array}{l}
\dot{v}_{c}(t)=\frac{1}{C}\left((1-u(t)) i_{l}(t)-i_{\varepsilon}(t)\right), \\
\dot{l}_{l}(t)=\frac{1}{L}\left(v_{\varepsilon}(t)-(1-u(t)) v_{c}(t)\right),
\end{array}\right.
$$

where $v_{\varepsilon}(t)=v_{e}(t)+v_{\text {loss }}(t)$ and $i_{\varepsilon}(t)=i(t)+i_{\text {loss }}(t)$. The signals $v_{\text {loss }}(t)$ and $i_{\text {loss }}(t)$ have been introduced to take into account the modeling errors (stray resistor and voltage drop across the IGBT type switch). Given the sources of the losses, $v_{\varepsilon}(t), v_{e}(t)$ and $i_{\varepsilon}(t), i(t)$ have the same nature, that is, step-like. The different signals are assumed to belong to the following bounded sets

$$
\begin{array}{r}
v_{c}(t) \in\left[v_{c_{\min }}, v_{c_{\max }}\right], \quad i_{l}(t) \in\left[i_{l_{\min }}, i_{l_{\max }}\right], \\
v_{\varepsilon}(t) \in\left[v_{\varepsilon_{\min }}, \quad v_{\varepsilon_{\max }}\right], \\
i_{\varepsilon}(t) \in\left[i_{\varepsilon_{\min }}, \quad i_{\varepsilon_{\max }}\right], \quad u(t) \in\left[u_{\min }, u_{\max }\right] .
\end{array}
$$

\section{Control algorithm}

In this section, the main theoretical development of the paper, namely a robust nonlinear state feedback control law for $v_{c}$ is presented. The proposed algorithm is an integral high gain type controller, which has already been theoretically developed in the literature (see [42]). It should be noted that the explicit incorporation of an integral action, into the synthesis model, is common, whether for linear or nonlinear approaches. Usually, the integral action is introduced after shifting the state space coordinates that leads to the Brunovsky canonical form, because it greatly simplifies the analysis. Indeed, most of the existing nonlinear control are synthesized for systems under this form since every uniformly controllable and observable systems can be put into this form [43]. But, in presence of model uncertainties, the inverse transformation that is necessarily done on-line to calculate the value of the control variable, may cause a loss of the integral action effects. To cope with this difficulty and to ensure robust static performances, even in presence of uncertainties, the integral action is directly introduced in the original coordinates. This, then, leads to the following extended synthesis model

$$
\left\{\begin{array}{l}
\dot{v}_{c}(t)=\frac{1}{C}\left((1-u(t)) i_{l}(t)-i_{\varepsilon}(t)\right), \\
\dot{i}_{l}(t)=\frac{1}{L}\left(v_{\varepsilon}(t)-(1-u(t)) v_{c}(t)\right), \\
\dot{u}(t)=\mu(t),
\end{array}\right.
$$

where $\mu(t)$ is the first derivative of the physical control variable $u(t)$, and becomes the virtual input of the system. Another advantage of this structure is that it allows to deal with the negative effects of the actuator saturation on the control variable. This can now be easily solved using a standard anti-windup structure [44].

The presentation of the proposed algorithm is split in three parts. Firstly, a nonlinear control law is designed in the nominal case, that is to say, when the supply voltage and the output current are constant and a priori known. Secondly, an observer is designed in order to deliver an estimation of the variables $v_{\varepsilon}(t)$ and $i_{\varepsilon}(t)$ using the available on line measurements, namely $v_{c}(t)$ and $i_{l}(t)$. Finally, the two previous parts are combined to obtain both a robust control law that takes into account the changes in the voltage supply and the load and a diagnostic tool for the monitoring of these variables, the proposed control law is illustrated on Fig. 2. 


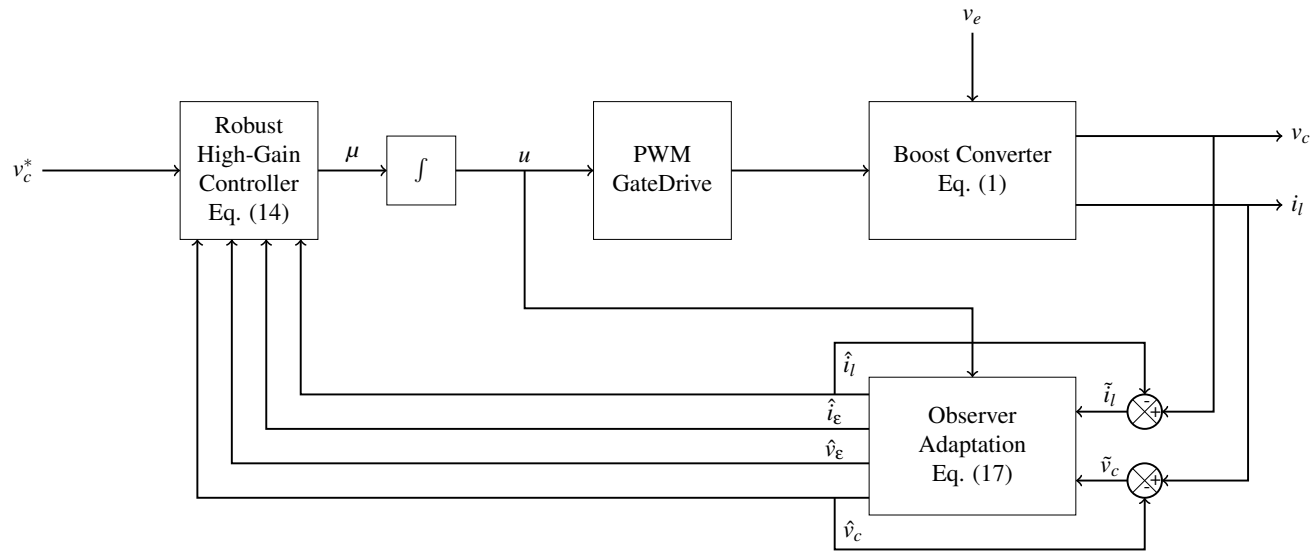

Fig. 2: Scheme of the control law implementation presented in section 3.3

\subsection{Nominal case}

In the nominal case, the supply voltage and the output current are constant and perfectly known. In this configuration, the power losses are equal to zero and system (3) can be written as:

$$
\left\{\begin{array}{l}
\dot{v}_{c}(t)=\frac{1}{C}\left((1-u(t)) i_{l}(t)-i_{\varepsilon}^{*}\right) \\
\dot{i}_{l}(t)=\frac{1}{L}\left(v_{\varepsilon}^{*}-(1-u(t)) v_{c}(t)\right) \\
\dot{u}(t)=\mu(t)
\end{array}\right.
$$

where $v_{\varepsilon}^{*}$ and $i_{\varepsilon}^{*}$ denote the nominal values of $v_{\varepsilon}(t)$ and $i_{\varepsilon}(t)$ respectively. The Brunovsky canonical form characterizes the class of nonlinear systems for which the nonlinearity has a triangular structure. For such a class of systems, the high-gain control law has been proposed in the literature using the duality with the high-gain type observer. The control law synthesis requires the existence of a diffeomorphism that puts the physical model into the Brunovsky form. For that purpose, let us consider the change of coordinates $z \triangleq \psi\left(v_{c}, i_{l}, u ; v_{\varepsilon}, i_{\varepsilon}\right)$ where $\psi$ is defined by

$$
\psi\left(v_{c}, i_{l}, u ; v_{\varepsilon}^{*}, i_{\varepsilon}^{*}\right)=\left(\begin{array}{c}
C v_{c}^{2}+L i_{l}^{2} \\
2\left(i_{l} v_{\varepsilon}^{*}-v_{c} c_{\varepsilon}^{*}\right) \\
2\left(\frac{v_{\varepsilon}^{* 2}}{L}+\frac{i_{\varepsilon}^{* 2}}{C}\right)-2(1-u)\left(\frac{v_{\varepsilon}^{*} v_{c}}{L}+\frac{i_{\varepsilon}^{*} i_{l}}{C}\right)
\end{array}\right),
$$

Using (5), the physical model (4) can be put under the following canonical form

$$
\left\{\begin{array}{l}
\dot{z}_{1}(t)=z_{2}(t) \\
\dot{z}_{2}(t)=z_{3}(t) \\
\dot{z}_{3}(t)=\bar{f}\left(z(t) ; v_{\varepsilon}^{*}, i_{\varepsilon}^{*}\right) \mu(t)+\bar{\phi}\left(z(t) ; v_{\varepsilon}^{*}, i_{\varepsilon}^{*}\right)
\end{array}\right.
$$

where $\bar{f}\left(z(t) ; v_{\varepsilon}^{*}, i_{\varepsilon}^{*}\right)$ and $\bar{\phi}\left(z(t) ; v_{\varepsilon}^{*}, i_{\varepsilon}^{*}\right)$ have the following expressions

$$
\begin{aligned}
\bar{f}\left(z ; v_{\varepsilon}^{*}, i_{\varepsilon}^{*}\right) & =f\left(\psi^{-1}\left(z ; v_{\varepsilon}, i_{\varepsilon}\right) ; v_{\varepsilon}^{*}, i_{\varepsilon}^{*}\right), \\
& =f\left(v_{c}, i_{l}, u ; v_{\varepsilon}^{*}, i_{\varepsilon}^{*}\right), \\
\bar{\phi}\left(z ; v_{\varepsilon}^{*}, i_{\varepsilon}^{*}\right) & =\phi\left(\psi^{-1}\left(z ; v_{\varepsilon}^{*}, i_{\varepsilon}^{*}\right) ; v_{\varepsilon}^{*}, i_{\varepsilon}^{*}\right), \\
& =\phi\left(v_{c}, i_{l}, u, v_{\varepsilon}^{*}, i_{\varepsilon}^{*}\right), \\
& =\frac{2}{L C}(u-1)^{2}\left(v_{c} i_{\varepsilon}^{*}-i_{l} v_{\varepsilon}^{*}\right) .
\end{aligned}
$$


with

$$
\begin{array}{r}
f\left(v_{c}, i_{l}, u ; v_{\varepsilon}^{*}, i_{\varepsilon}^{*}\right)=2\left(\frac{v_{\varepsilon}^{*} v_{c}}{L}+\frac{i_{\varepsilon}^{*} i_{l}}{C}\right), \\
\phi\left(v_{c}, i_{l}, u, v_{\varepsilon}^{*}, i_{\varepsilon}^{*}\right)=\left(\frac{2}{L C}(u-1)^{2}\left(v_{c} i_{\varepsilon}^{*}-i_{l} v_{\varepsilon}^{*}\right)\right),
\end{array}
$$

The nominal values $v_{\varepsilon}^{*}$ and $i_{\varepsilon}^{*}$ are considered as parameters, hence, the function $\psi$ is seen as a function from $\mathbb{R}^{3}$ to $\mathbb{R}^{3}$, and $\psi^{-1}\left(z ; v_{\varepsilon}^{*}, i_{\varepsilon}^{*}\right)$ denotes the function verifying:

$$
\psi^{-1}\left(\psi\left(v_{c}, i_{l}, u ; v_{\varepsilon}^{*}, i_{\varepsilon}^{*}\right) ; v_{\varepsilon}^{*}, i_{\varepsilon}^{*}\right)=\left(v_{c}, i_{l}, u\right)
$$

for all $\left(v_{c} ; i_{l}, u\right)$ in $\mathbb{R}^{3}$.

The control objective can be expressed in this new basis as: the state vector $z(t)$ must be maintained in the vicinity of the following constant reference vector $z^{*} \in \mathbb{R}^{3}$

$$
z^{*}=\left(\begin{array}{c}
C\left(v_{c}^{*}\right)^{2}+L\left(i_{l}^{*}\right)^{2} \\
0 \\
0
\end{array}\right)
$$

For the class of systems (6), the following high-gain type control law is proposed:

$$
\mu(t)=-\frac{1}{f\left(v_{c}, i_{l}, u ; v_{\varepsilon}^{*}, i_{\varepsilon}^{*}\right)} \times k_{c}\left[\lambda^{3}, 3 \lambda^{2}, 3 \lambda\right] \times\left(z-z^{*}\right)
$$

where $k_{c}>0, \lambda>0$ are synthesis parameters. The control law can now be expressed using the physical variables

$$
\mu(t)=-\frac{1}{f\left(v_{c}, i_{l}, u ; v_{\varepsilon}^{*}, i_{\varepsilon}^{*}\right)} \mathrm{v},
$$

where $v$ is given by

$$
v=k_{c}\left[\lambda^{3}, 3 \lambda^{2}, 3 \lambda\right] \times\left(\psi\left(v_{c}, i_{l}, u ; v_{\varepsilon}^{*}, i_{\varepsilon}^{*}\right)-\psi\left(v_{c}^{*}, i_{l}^{*}, u^{*} ; v_{\varepsilon}^{*}, i_{\varepsilon}^{*}\right)\right),
$$

Semicolons are used in the arguments of the different functions to enhance the fact that $\left(v_{c}, i_{l}, u\right)$ are the main variables while $v_{\varepsilon}^{*}$ and $i_{\varepsilon}^{*}$ are only parameters.

The nominal values of $i_{l}$ and $u$, are denoted $i_{l}^{*}$ and $u^{*}$, and can be obtained by replacing their expressions in (4):

$$
i_{l}^{*}=\frac{i_{\varepsilon}^{*}}{v_{\varepsilon}^{*}} v_{c}^{*}, \quad u^{*}=1-\frac{v_{\varepsilon}^{*}}{v_{c}^{*}} .
$$

One has the following result of convergence.

Proposition 1. If $k_{c}$ and $\lambda$ are chosen sufficiently large, then the control law (14)-(15) ensures that the tracking errors $\tilde{v}_{c}(t)=v_{c}(t)-v_{c}^{*}, \tilde{i}_{l}(t)=i_{l}(t)-i_{l}^{*}$ and $\tilde{u}(t)=u(t)-u^{*}$ converge to zero where $v_{c}(t), i_{l}(t)$ and $u(t)$ are the solutions of equation (4), $v_{c}^{*}$ is the specified output voltage, $i_{l}^{*}$ and $u^{*}$ are given by (16).

Remark 1. The control law has been designed for the system in the Brunovsky form and is made up of the product of a linear high-gain state feedback and a nonlinear term. The nonlinear term is used in order to compensate the nonlinearity $\bar{f}$ and is associated to the parameter $k_{c}$. When $\bar{f}$ is perfectly known, it is sufficient to take $k_{c}>1 / 2$. The linear part allows to 
place all the eigenvalues of the linear part of the closed loop system at $-\lambda$. Then $\lambda$ is closely related to the closed loop time constant. Moreover, $\lambda$ has to be greater than a lower bound in order to dominate the nonlinearity $\bar{\phi}$. This lower bound is proportional to the Lipschitz constant of $\bar{\phi}$.

It is worth noting that the different variables are bounded (see (2)), thus, if $\lambda$ is taken too large, the overshoot may lead to a saturation.

Proof. See section A in the appendix.

Now let us consider the more realistic case where the converter is not perfect $\left(v_{\text {loss }} \neq 0, i_{\text {loss }} \neq 0\right)$ and the supply voltage and the load are unknown but piecewise constant. In that case, the expression of the function $f$ can be deduced from (3) and it depends on the exact values of $v_{\varepsilon}(t)$ and $i_{\varepsilon}(t)$ instead of their nominal values. As a consequence, the control law (14) will not ensure exact feedback linearization and the closed loop dynamical performances will be degraded. To overcome this problem, a nonlinear observer is synthesized in the next section to give an estimation of $v_{\varepsilon}(t)$ and $i_{\varepsilon}(t)$ and thus of $f$. These estimations can also be useful for diagnostic purposes and then achieve the second objective of the proposed monitoring algorithm.

\subsection{Observer for the perturbation}

An observer is designed in this section to estimate $v_{\varepsilon}(t)$ and $i_{\varepsilon}(t)$ in the case where the supply voltage $v_{e}(t)$ and the output current $i(t)$ changes are piecewise constant. These estimates can be used to get useful informations about the state of charge of the energy storage component which supplies the DC/DC converter [45]. Since the signals $i_{\varepsilon}(t)$ and $v_{\varepsilon}(t)$ are piecewise constants and the measured signals are $v_{c}(t)$ and $i_{l}(t)$, an observer, based on [46] is given by

$$
\left\{\begin{array}{l}
\dot{\hat{v}}_{c}(t)=\frac{1}{C}\left((1-u(t)) \hat{i}_{l}(t)-\hat{i}_{\varepsilon}(t)\right)-2 \theta\left(\hat{v}_{c}(t)-v_{c}(t)\right), \\
\hat{i}_{l}(t)=\frac{1}{L}\left(\hat{v}_{\varepsilon}(t)-(1-u(t)) \hat{v}_{c}(t)\right)-2 \theta\left(\hat{i}_{l}(t)-i_{l}(t)\right), \\
\hat{i}_{\varepsilon}(t)=C \theta^{2}\left(\hat{v}_{c}(t)-v_{c}(t)\right) \\
\hat{\hat{v}}_{\varepsilon}(t)=-L \theta^{2}\left(\hat{i}_{l}(t)-i_{l}(t)\right)
\end{array}\right.
$$

where $\theta>1$ is the sole observer design parameter.

Proposition 2. Assume that the input $u(t)$ belongs to a bounded set $\mathcal{U}$ for all $t>0$, then there exists $\theta_{0}>1$ such that for all $\theta>\theta_{0}$ the observation error converges asymptotically to zero, independently of the control signal, as follows:

$$
\exists \theta_{0}>0, \quad \forall \theta>\theta_{0}, \exists \alpha>0, \exists \mu_{\theta}>0, \forall u \in \mathcal{U}, \forall \hat{x}(0) \in \mathbb{R}^{4},
$$

such that

$$
\|\hat{x}(t)-x(t)\|<\alpha e^{-\mu_{\theta} t}\|\hat{x}(0)-x(0)\|,
$$

where $\lim _{\theta \rightarrow+\infty} \mu_{\theta}=+\infty$, with

$$
x=\left[v_{c}, i_{l}, i_{\varepsilon}, v_{\varepsilon}\right]^{T} \text {, and } \hat{x}=\left[\hat{v}_{c}, \hat{i}_{l}, \hat{i}_{\varepsilon}, \hat{v}_{\varepsilon}\right]^{T} .
$$

Remark 2. As for the control law, the eigenvalues of the linear part of the observer error system are located at $-\theta$. This design parameter must be greater than a lower bound $\theta_{0}$, which is proportional to the Lipschitz constant of the nonlinear part.

Proof. See section B in the appendix.

Now that estimations of $v_{\varepsilon}$ and $i_{\varepsilon}$ are available, an estimation $f_{e}\left(v_{c}, i_{l}, u ; \hat{v}_{\varepsilon}, \hat{i}_{\varepsilon}\right)$ of $f\left(v_{c}, i_{l}, u ; v_{\varepsilon}, i_{\varepsilon}\right)$ is given as follows:

$$
f_{e}\left(v_{c}, i_{l}, u ; \hat{v}_{\varepsilon}, \hat{i}_{\varepsilon}\right)=2\left(\frac{v_{c} \operatorname{sat}_{1}\left(\hat{v}_{\varepsilon}\right)}{L}+\frac{i_{l} \operatorname{sat}_{2}\left(\hat{i}_{\varepsilon}\right)}{C}\right)
$$


with

$$
\begin{aligned}
& \operatorname{sat}_{1}(\sigma)= \begin{cases}v_{\varepsilon_{\min }} & \text { if } \sigma<v_{\varepsilon_{\min }} \\
\sigma & \text { if } \sigma \in\left[v_{\varepsilon_{\min }}, v_{\varepsilon_{\max }}\right], \\
v_{\varepsilon_{\max }} & \text { if } \sigma>v_{\varepsilon_{\max }}\end{cases} \\
& \operatorname{sat}_{2}(\sigma)= \begin{cases}i_{\varepsilon_{\min }} & \text { if } \sigma<i_{\varepsilon_{\min }} \\
\sigma & \text { if } \sigma \in\left[i_{\varepsilon_{\min }}\right. \\
i_{\varepsilon_{\max }} & \text { if } \left.\sigma>i_{\varepsilon_{\max }}\right] .\end{cases}
\end{aligned}
$$

The saturating functions $\operatorname{sat}_{1}(\sigma)$ and $\operatorname{sat}_{2}(\sigma)$ have been introduced to ensure both that $f_{e}\left(v_{c}, i_{l}, u ; \hat{v}_{\varepsilon}, \hat{i}_{\varepsilon}\right)$ is a positive function, during the observer transient state and that $f\left(v_{c}, i_{l}, u, v_{\varepsilon}, i_{\varepsilon}\right)-f_{e}\left(v_{c}, i_{l}, u ; \hat{v}_{\varepsilon}, \hat{i}_{\varepsilon}\right)$ converges to zero when $v_{\varepsilon}(t)$ and $i_{\varepsilon}(t)$ are constant [47].

\subsection{Robust control law}

In the non-nominal case, all the nominal values used in the control law equations (5)-(14)-(15)-(16) are replaced by the estimates of the corresponding variables. The scheme for the implementation of the control law is depicted on Fig. 2 . The estimations of $v_{\varepsilon}(t)$ and $i_{\varepsilon}(t)$ can be directly obtained from observer (17) whereas (16) is replaced by :

$$
\hat{i}_{l}^{*}(t)=\frac{\hat{i}_{\varepsilon}(t)}{\hat{v}_{\varepsilon}(t)} v_{c}^{*}, \quad \hat{u}^{*}(t)=1-\frac{\hat{v}_{\varepsilon}(t)}{v_{c}^{*}}
$$

Using, in addition, the estimation $f_{e}\left(v_{c}, i_{l}, u ; \hat{v}_{\varepsilon}, \hat{i}_{\varepsilon}\right)$ delivered by (19), the state feedback (14)-(5) can be modified as follows:

$$
\mu=-\frac{1}{f_{e}\left(v_{c}, i_{l}, u ; \hat{v}_{\varepsilon}, \hat{i}_{\varepsilon}\right)} \hat{v}
$$

and

$$
\hat{\mathrm{v}}=k_{c}\left[\lambda^{3}, 3 \lambda^{2}, 3 \lambda\right] \times\left(\psi\left(v_{c}, i_{l}, u ; s a t_{1}\left(\hat{v}_{\varepsilon}\right), s a t_{2}\left(\hat{i}_{\varepsilon}\right)\right)\right.
$$

$$
\left.-\psi\left(v_{c}^{*}, \hat{i}_{l}^{*}, \hat{u}^{*} ; \operatorname{sat}_{1}\left(\hat{v}_{\varepsilon}\right), \operatorname{sat}_{2}\left(\hat{i}_{\varepsilon}\right)\right)\right) .
$$

The main convergence result of the paper is stated below.

Proposition 3. The control law (17), (19), (22), (23) ensures that $v_{c}(t)$, the solution of system (3), converges exponentially toward $v_{c}^{*}$ provided that $\theta, k_{c}$ and $\lambda$ are chosen sufficiently large.

Remark 3. The sole difference for the tunning of the design parameters compared to the nominal part concerns $k_{c}$. Indeed, in the non nominal case, the multiplicative nonlinearity $\bar{f}$ is no longer exactly compensated. The parameter $k_{c}$ is $u s e d$ in order to handle this issue and has to satisfy $k_{c}>\frac{f_{\min }}{2 f_{\max }}$ where $f_{\min }$ and $f_{\max }$ are respectively the lower and upper bounds of $f$.

Proof. See section $\mathrm{C}$ in the appendix.

Remark 4. The conditions (51) and (56), established in appendix $C$, which ensure the convergence of the control algorithm may be very restrictive, since they provide a lower bound for the synthesis parameters $k_{c}$ and $\lambda$. Nevertheless, many simulations tests have shown that the proposed control law performs well for synthesis parameters values that are lower than the theoretical bounds.

\section{Applications}

The performances of the proposed state feedback control law have been firstly validated with simulations on Matlab/Simulink environment and compared to those obtained with a standard current mode controller [7]. For that purpose, a realistic boost converter simulator has been created to reproduce as closely as possible the behavior of the continuous system. Several stray inductors, resistors and capacitors associated to the connecting tracks, cables and semiconductor devices have 
been introduced. The coil is highly nonlinear and displays hysteric behavior due to the magnetic cycle of its core. At usual operating points, effects of these phenomena and of the stray components are however mostly negligible. The IGBT has been considered as an ideal switch. Finally, the imperfections that have been taken into account in our simulation of the average model are the stray resistor of the coil and the saturation of the coil current. Please note that these operating points have usually been chosen in the literature in the more binding cases [17] and then gave us a satisfying benchmark test for the actual proposed control law. Table 1 reports the simulation parameters values which are the same than the experimental ones, presented next.

\begin{tabular}{cccc}
\hline \hline Symbol & Quantity & Value & Unity \\
\hline$L$ & Coil & 2 & $\mathrm{mH}$ \\
$C$ & Capacitor & 6.8 & $\mathrm{mF}$ \\
$R_{L}$ & Resistor & 8 & $\mathrm{~m} \Omega$ \\
$R_{e s r}$ & Resistor & 2.5 & $\mathrm{~m} \Omega$ \\
$u_{\max }$ & Maximum control value & 0.98 & - \\
$u_{\min }$ & Minimum control value & 0.02 & - \\
$v_{e}^{*}$ & Nominal battery voltage & 12 & Volts \\
$v_{c}^{*}$ & Reference output voltage & 24 & Volts \\
& PWM switch frequency & 20 & $\mathrm{kHz}$ \\
\hline
\end{tabular}

Table 1: Experimental parameters values

\subsection{Simulation results}

First, let us examine the nonlinear control law design. Many design softwares are now available for the synthesis of linear control laws whereas this is not yet the case for nonlinear control schemes where the choice of the design parameters values is often a difficult task. The proposed control law gives a great answer to this challenge because the parameter values are readily determinable. Indeed, as long as $k_{c}$ remains constant, $\theta$ and $\lambda$ directly determine the values of the poles of the linear parts of the observer and of the state feedback respectively. The Fig. 3 shows the transient responses when the load switches from $R=5 \Omega$ to $R=30 \Omega$ at $75 \mathrm{~ms}$ and switches back to $R=5 \Omega$ at $150 \mathrm{~ms}$ for different values of $\theta$ and $\lambda$. The relationship between the values of these parameters and the response time is clearly shown there. Subject to compliance with conditions (18) and (56) an increase in the values of $\theta$ and $\lambda$ brings a better disturbance rejection but increases the closed loop sensitivity with respect to measurement noise.

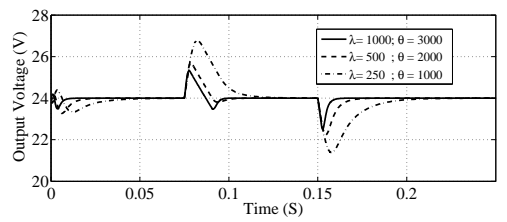

Fig. 3: Simulated transient of the boost converter under a load step variation $(R=5 \Omega \rightarrow R=30 \Omega \rightarrow R=5 \Omega)$ with different parameter values of the nonlinear controller .

To determine the best values of the synthesis parameters for the control law (22) with regard to the input/output closed loop performances, many simulations have been carried out using the previous simulator. The obtained values are:

$$
\theta=2000, \quad \lambda=500, \quad k_{c}=1 .
$$


The performances of the control law (22) with the previous design parameters values have been evaluated in many configurations. For comparison purposes, a current mode controller has been designed. It was formed by a peak current modulator associated with a voltage control loop using a PI controller. This control structure is commonly used for static converters in the literature. A comparison of both algorithms allows us to quantify the performances of the nonlinear control law. The PI controller has been designed using the nominal average model and with specifications that guarantee a $60^{\circ}$ phase margin and a $10 d B$ gain margin which are common specifications in power electronic [48].

A first experiment was conducted where the load changes from $R=5 \Omega$ to $R=30 \Omega$ and changes back to $R=5 \Omega$ with two operating point duty cycle values, respectively $d=0.5$ (nominal value, Fig. 4a) and $d=0.7$ (non-nominal value, Fig. 4b). Then, the performances of the control scheme have been tested in the presence of supply voltage variations (Fig. 4c). The step like corresponding profile consists of a change in level from $v_{e}=12 \mathrm{~V}$ to $v_{e}=7 \mathrm{~V}$ at $t=100 \mathrm{~ms}$ and again to $v_{e}=12 \mathrm{~V}$ at $t=200 \mathrm{~ms}$.

The nonlinear control law obviously satisfies the transient and steady state performances requirements for both operating point duty cycles, while the current mode controller does not ensure the stability of the closed loop when working too far from the nominal operating point. Moreover, the nonlinear approach obviously gives the best settling time in the nominal case. It is worth pointing out that the converter, with the nonlinear algorithm, enters in discontinuous mode on figure $4 \mathrm{~b}$ between $t=0.11 \mathrm{~s}$ and $t=0.15 \mathrm{~s}$. The robustness of our algorithm with respect to the change between continuous and discontinuous mode is due to the saturation functions which have been applied directly to the estimates provided by the observer (19).
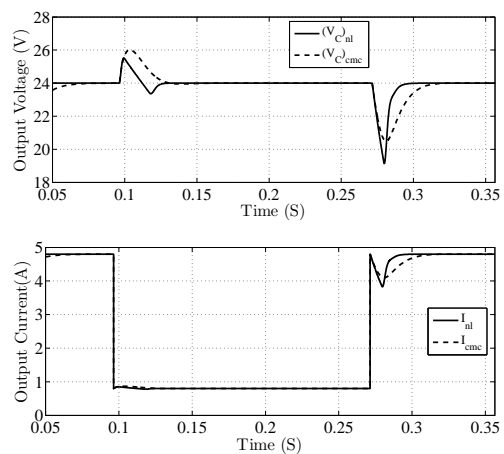

(a) load step transient $(R: 5 \Omega \rightarrow 30 \Omega \rightarrow 5 \Omega$ ) in nominal point $d=0.5$.
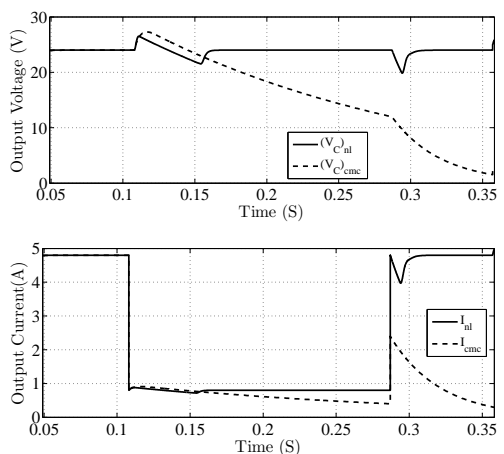

(b) load step transient $(R: 5 \Omega \rightarrow 30 \Omega \rightarrow 5 \Omega)$ in nominal point $d=0.7$.
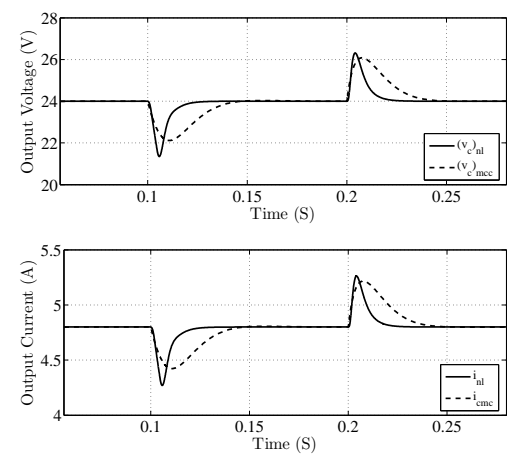

(c) supply voltage step transient $\left(v_{e}: 12 \mathrm{~V} \rightarrow\right.$ $7 \mathrm{~V} \rightarrow 12 \mathrm{~V})$

Fig. 4: Simulated transient of the boost converter with nonlinear controller (solid line) and current-mode controller (dashed line).

Let us now examine the observer performances. This observer has first been designed to deliver an estimation of the supply voltage and the output current for monitoring purposes using the available measurements, namely, the output voltage and the coil current. An experiment has been conducted where the supply voltage changes from $v_{e}=12 \mathrm{~V}$ to $v_{e}=7 \mathrm{~V}$ at $t=250 \mathrm{~ms}$ and again to $v_{e}=12 \mathrm{~V}$ at $t=550 \mathrm{~ms}$ (Fig. 5c). Moreover, for each supply voltage level, a load profile has been specified corresponding to a change from $R=5 \Omega$ to $R=30 \Omega$ and again to $R=5 \Omega$ (F $5 \mathrm{~d}$ ). Finally, a step-like output disturbance has been applied at $t=650 \mathrm{~ms}$ in order to simulate an offset on the sensor measurement. The observer performances are represented on Fig. 5a, 5b, 5c and 5d.

The convergence of the observer is clearly ensured for all the configurations that have been tested thanks to the specified disturbance profiles. The output voltage and the coil current are correctly estimated without any bias during all the steady state phases. A bias appears in the estimation of the supply voltage and the output current which can be easily explained by the model uncertainties that have been taken into account. In fact, the observer delivers estimations of both $v_{\varepsilon}(t)$ and $i_{\varepsilon}(t)$ instead of $v_{e}(t)$ and $i(t)$ respectively. In the actual simulation case, the difference between the previous signals is directly connected to the coil resistor that has been introduced in the simulator. A particular point needs to be emphasized on Fig. $5 \mathrm{c}$ and $5 \mathrm{~d}$. The sensor offset is responsible for an additional bias on the supply voltage and output current estimates. This test shows that the observer can be used to replace the sensors or to provide an oversight of the converter. Indeed, since $v_{e}(t)$ and $i(t)$ are constants, the differences between the measured and estimated signals are constant. A tabulation of the latter according to the variables can be used to measure the aging deviation of the components constituting the converter. 


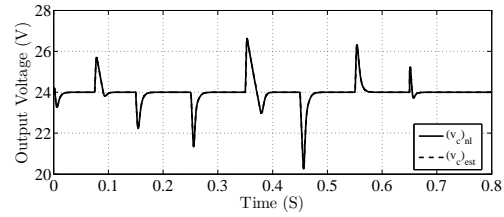

(a) Estimation of $v_{c}(t)$

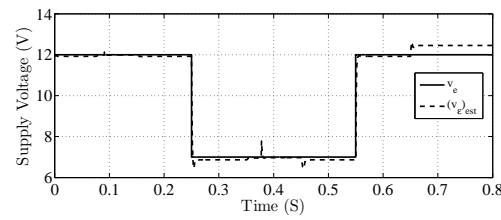

(c) Estimation of $v_{\varepsilon}(t)$

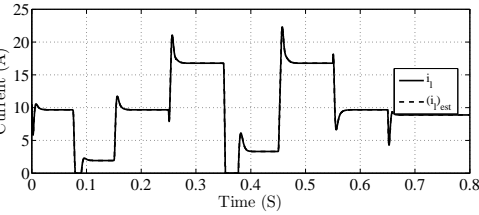

(b) Estimation of $i_{l}(t)$

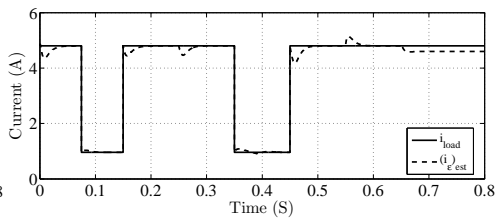

(d) Estimation of $i_{\varepsilon}(t)$

Fig. 5: Simulated state variables (solid lines) and estimated state variables (dashed lines)

\subsection{Experimental results}

The proposed nonlinear control law has been tested on an existing DC/DC Converter. The experimental setup is composed of an Insulate Gate Bipolar Transistor (IGBT) power switches, a capacitor and an inductor. The physical parameter values are given in Table 1. This experiment was conducted with a sampling period of $0.75 \mathrm{~ms}$, which is compatible with the imposed PWM switch frequency $\left(20 \mathrm{kHz}\right.$ ), and the reference output voltage $v_{c}^{*}$ has been set to $24 \mathrm{~V}$. Starting from the values (24) obtained in simulation with MATLAB/Simulink, the best input/output performances have been obtained with the following values:

$$
\theta=2000, \quad \lambda=1500, \quad k_{c}=1 .
$$

The empiric rule for the choice of the parameters $\lambda$ and $\theta$ with respect to the sampling period is to have $T_{s} \lambda_{\max } \approx 1$ and $T_{s} \theta_{\max } \approx 1[49,50]$. Some new approaches allowing to overrule these limitation have recently been proposed in [51].

The control algorithm has then been implemented using the 1102 Dspace Environment. One can notice that the voltage and, especially, the current measurements are very noisy, indeed any anti-aliasing filters has been used. It is particularly visible on Fig. 6d, where the stabilized supply voltage measurements are shown.

The goal of this experiment is to show that our control law can keep the output voltage to the reference value even in presence of changes in load and supply voltage, all the results are presented on Fig. 6. The changes in the load and the supply voltage have been applied manually to the converter and can be seen on Fig. $6 \mathrm{c}$ and $6 \mathrm{~d}$.

The estimates of $v_{\varepsilon}(t)$ and $i_{\varepsilon}(t)$ are depicted on Fig. $6 \mathrm{c}$ and $6 \mathrm{~d}$. The difference between $v_{e}(t)$ and $v_{\varepsilon}(t)$ clearly illustrates the power stage losses.

Similarly, the imposed variations of the output current are well estimated by the observer (see Fig. 6c). As shown in Fig. $6 a$, the regulation is offset position error free despite modeling errors, variations in supply voltage and load. The control objective is achieved with a slightly noisy input signal (Fig. 6e). The nonlinear process behavior is visible with the increase of noise on the output and input during the variation of the supply voltage $(3.2 s-4.5 s)$. The load variations cause a change in the power loss which results in an increase of the average value of the input signal (around 6.2s). There is no important peak in the value of the control variables to compensate the absence of knowledge of the uncertain parameters. This allows to avoid saturation of the coil which would lead to a magnetic saturation and thus to a nonlinear behavior of the physical system, as shown on Fig. 6b.

\section{Conclusion}

In this paper, a nonlinear approach for controlling a static boost converter has been presented, with several improvements with respect to the previous approaches. The underlying control system ensures an offset free performance even in presence of uncertain parameters. Such a feature is achieved by a cancellation of the effect of a nonlinear term acting on the input which is appropriately estimated using a nonlinear high-gain observer. Three features are worth to be mentioned. Firstly, the involved estimates are of importance because they can be used for supervision purposes for batteries or super-capacitors [45]. Secondly, the implementation of the control law has been greatly simplified as the integral action is implemented directly in physical coordinates. And finally, The convergence has been proven and validated by probing experimental results. 


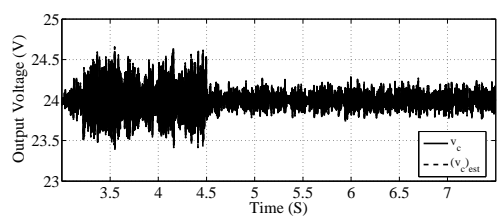

(a) Output performance $v_{c}(t)$ and $\hat{v}_{c}(t)$

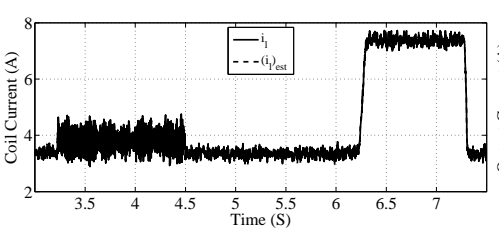

(b) Coil current measurement $i_{l}(t)$ and $\hat{i}_{l}(t)$

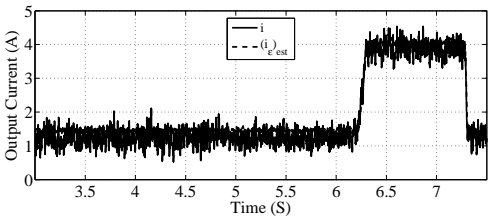

(c) Estimated current $i(t)$ and $\hat{i}_{\varepsilon}(t)$

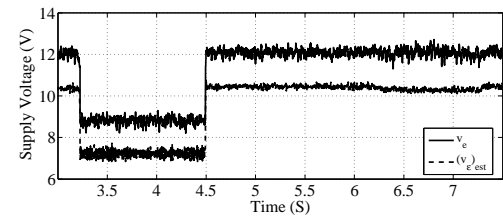

(d) Experimental Battery voltage value $v_{e}(t)$ and $\hat{v}_{\varepsilon}(t)$ estimated value

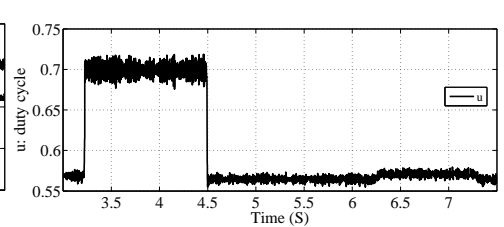

(e) Input experimental performance $u(t)$

Fig. 6: Experimental results.

\section{A Proof of proposition 1}

In the proof, for the sake of clarity, the dependence on the parameters $v_{\varepsilon}^{*}$ and $i_{\varepsilon}^{*}$ will be omitted, that is, the functions $\bar{f}$ and $\bar{\phi}$ will be denoted $\bar{f}(z)=\bar{f}\left(z ; v_{\varepsilon}^{*}, i_{\varepsilon}^{*}\right)$ and $\bar{\phi}(z)=\bar{\phi}\left(z ; v_{\varepsilon}^{*}, i_{\varepsilon}^{*}\right)$.

It is of particular importance to notice that the function $\bar{f}(z)$ is positive on the physical working subspace because of the bound on the physical signals given by (2). Moreover, the function $\bar{\phi}(z)$ is Lipschitz in its arguments over the domain of interest, since it is continuously differentiable and its arguments stay inside a bounded set, because of (2).

The state vector $z(t)$ must be maintained in the vicinity of the constant reference vector $v^{*}$. This is equivalent to

$$
\lim _{t \rightarrow+\infty} \tilde{z}(t)=0
$$

where $\tilde{z}(t)$ is the tracking error defined by

$$
\tilde{z}(t)=z(t)-z^{*}
$$

Using (6), (12) and (27), the control model can be written in the following Brunovsky triangular canonical form

$$
\dot{\tilde{z}}(t)=A_{c} \tilde{z}(t)+B_{c} \bar{f}(z(t)) \mu(t)+\bar{\varphi}(z(t)),
$$

with

$$
\begin{gathered}
A_{c}=\left(\begin{array}{cc}
0_{2 \times 1} & I_{2 \times 2} \\
0 & 0_{1 \times 2}
\end{array}\right), \quad B_{c}=\left(\begin{array}{c}
0_{2 \times 1} \\
1
\end{array}\right), \\
\bar{\varphi}(z)=\left(\begin{array}{c}
0_{2 \times 1} \\
\bar{\phi}(z)
\end{array}\right),
\end{gathered}
$$

where $I_{2 \times 2}, 0_{1 \times 2}$ and $0_{2 \times 1}$ are respectively the $2 \times 2$ identity matrix, the zero matrix of dimension $1 \times 2$ and the zero matrix of dimension $2 \times 1$.

The control law

$$
\mu(t)=-\frac{1}{\bar{f}(z(t))} v(\tilde{z}(t))
$$


provides an exponential convergence to zero of the tracking error for a sufficiently high value of the parameter synthesis $\lambda$, according to Theorem 3.2 in [42], where

$$
\mathrm{v}(\tilde{z}(t))=k_{c} B_{c}^{T} \bar{S} \Gamma_{\lambda} \tilde{z}(t)
$$

$k_{c}$ is a synthesis parameter, $\Gamma_{\lambda}$ is the following diagonal matrix

$$
\Gamma_{\lambda}=\operatorname{diag}\left(\lambda^{3}, \lambda^{2}, \lambda\right)
$$

and the matrix $\bar{S}$ is the positive definite solution of the following algebraic Lyapunov equation:

$$
\bar{S}+A_{c}^{T} \bar{S}+\bar{S} A_{c}=\bar{S} B_{c} B_{c}^{T} \bar{S}
$$

This choice for the gain is a direct transposition of the high-gain observer from [52] exploiting the duality between control and observation. Let $B_{c}^{T}=C_{c}$, since the algebraic Lyapunov equation $S+A_{c}^{T} S+S A_{c}=C_{c} C_{c}^{T}$ has a unique symmetric positive definite solution $S$, one can deduce that equation (33) has a unique symmetric positive definite solution $\bar{S}=T S^{-1} T$ with

$$
T=\left(\begin{array}{lll}
0 & 0 & 1 \\
0 & 1 & 0 \\
1 & 0 & 0
\end{array}\right) .
$$

With this choice for the Lyapunov equation, one has $B_{c}^{T} \bar{S}=C S^{-1} T=\left[\begin{array}{lll}1 & 3 & 3\end{array}\right]$. This implies that all the eigenvalue of the matrix $A_{c}-B_{c} B_{c}^{T} \bar{S} \Gamma_{\lambda}$ are equal to $-\lambda$, this simplifies the implementation and the proof of convergence without introducing further constraints on the control performance.

In the original coordinates, the controller (30) is given by (14)-(5), which ends the proof.

\section{B Proof of proposition 2}

Our system can be written into the following form (studied in [46])

$$
\left\{\begin{array}{l}
\dot{x}(t)=A_{o} x(t)+\varphi(x(t), u(t)), \\
y=C_{o} x(t)
\end{array}\right.
$$

where $u(t)$ is the control variable, $x(t)$ is the state of system (34), defined by

$$
x(t)=\left[\begin{array}{l}
x^{1}(t) \\
x^{2}(t)
\end{array}\right] \in \mathbb{R}^{4}
$$

with

$$
\begin{gathered}
x^{1}(t)=\left[\begin{array}{l}
x_{1}^{1}(t) \\
x_{2}^{1}(t)
\end{array}\right]=\left[\begin{array}{c}
v_{c}(t) \\
i_{l}(t)
\end{array}\right], \\
x^{2}(t)=\left[\begin{array}{l}
x_{1}^{2}(t) \\
x_{2}^{2}(t)
\end{array}\right]=\left[\begin{array}{c}
-i_{\varepsilon}(t) / C \\
v_{\varepsilon}(t) / L
\end{array}\right],
\end{gathered}
$$

and the matrices $A_{o}$ and $C_{o}$ are defined by

$$
A_{o}=\left[\begin{array}{ll}
0_{2 \times 2} & I_{2 \times 2} \\
0_{2 \times 2} & 0_{2 \times 2}
\end{array}\right], \quad C_{o}=\left[\begin{array}{ll}
I_{2 \times 2} & 0_{2 \times 2}
\end{array}\right]
$$


The function $\varphi(x(t), u(t))$ is globally Lipschitz with respect to $x(t)$ uniformly in $u(t)$ and is given by

$$
\varphi(x(t), u(t))=\left[\begin{array}{l}
\varphi^{1}(x(t), u(t)) \\
\varphi^{2}(x(t), u(t))
\end{array}\right]
$$

with

$$
\begin{gathered}
\varphi^{1}(x(t), u(t))=\left[\begin{array}{c}
(1-u(t)) x_{2}^{1}(t) / C \\
-(1-u(t)) x_{1}^{1}(t) / L
\end{array}\right], \\
\varphi^{2}(x(t), u(t))=\left[\begin{array}{c}
0_{2 \times 1} \\
0_{2 \times 1}
\end{array}\right] .
\end{gathered}
$$

Lemma 2.1 in [46] can be applied, since $v_{\varepsilon}(t)$ and $i_{\varepsilon}(t)$ are supposed piecewise constant, hence ensuring exponential convergence of the estimation error on every time interval where $v_{\varepsilon}(t)$ and $i_{\varepsilon}(t)$ are constants.

\section{Proof of proposition 3}

As in the proof of proposition 1, the system (3) is first transformed into the Brunovsky canonical form (6) through the change of coordinates $z=\psi\left(v_{c}, i_{l}, u ; v_{\varepsilon}, i_{\varepsilon}\right)$ :

$$
\left\{\begin{array}{l}
\dot{z}_{1}(t)=z_{2}(t) \\
\dot{z}_{2}(t)=z_{3}(t) \\
\dot{z}_{3}(t)=\bar{f}\left(z(t) ; v_{\varepsilon}, i_{\varepsilon}\right) \mu(t)+\bar{\phi}\left(z(t) ; v_{\varepsilon}, i_{\varepsilon}\right),
\end{array}\right.
$$

where $\bar{f}$ and $\bar{\phi}$ are defined as in (7) and (9) respectively. For the sake of clarity, the notation $\bar{f}(z)$ and $\bar{\phi}(z)$ is considered instead of $\bar{f}\left(z ; v_{\varepsilon}, i_{\varepsilon}\right)$ and $\bar{\phi}\left(z ; v_{\varepsilon}, i_{\varepsilon}\right)$ in the rest of the proof.

The reference trajectory, is no longer constant in the non-nominal case, it is given by

$$
\begin{aligned}
z^{*}(t) & =\psi\left(v_{c}^{*}, i_{l}^{*}(t), u^{*}(t), v_{\varepsilon}(t), i_{\varepsilon}(t)\right), \\
& =\psi\left(v_{c}^{*}, \frac{i_{\varepsilon}(t)}{v_{\varepsilon}(t)} v_{c}^{*}, 1-\frac{v_{\varepsilon}(t)}{v_{c}^{*}}, v_{\varepsilon}(t), i_{\varepsilon}(t)\right), \\
& \triangleq \kappa\left(v_{c}^{*}, v_{\varepsilon}(t), i_{\varepsilon}(t)\right) .
\end{aligned}
$$

Similarly, the estimate of the reference trajectory $z^{*}(t)$ is given by

$$
\begin{aligned}
\hat{z}^{*}(t) & =\psi\left(v_{c}^{*}, \hat{i}_{l}^{*}(t), \hat{u}^{*}(t), \operatorname{sat}_{1}\left(\hat{v}_{\varepsilon}(t)\right), \operatorname{sat}_{2}\left(\hat{i}_{\varepsilon}(t)\right)\right), \\
& =\kappa\left(v_{c}^{*}, \operatorname{sat}_{1}\left(\hat{v}_{\varepsilon}(t)\right), \operatorname{sat}_{2}\left(\hat{i}_{\varepsilon}(t)\right)\right) \\
& =\left(\begin{array}{c}
C\left(v_{c}^{*}\right)^{2}+L\left(\frac{\operatorname{sat}_{2}\left(\hat{i}_{\hat{\varepsilon}}(t)\right)}{\operatorname{sat}_{1}\left(\hat{v}_{\varepsilon}(t)\right)} v_{c}^{*}\right)^{2} \\
0 \\
0
\end{array}\right)
\end{aligned}
$$

where the function $s a t_{1}$ and $s a t_{2}$ are define in (20).

The aim of this proof, is to show that the error $\tilde{z}(t)=z(t)-z^{*}(t)$ converges exponentially to zero on any time interval where $z^{*}(t)$ is constant. Note that saying that $z^{*}(t)$ is constant is equivalent to say that $v_{\varepsilon}(t)$ and $i_{\varepsilon}(t)$ are constant since $z^{*}(t)$ is a function of $v_{c}^{*}, v_{\varepsilon}$ and $i_{\varepsilon}$.

In order to do that, it is sufficient to prove that the next two signals

$$
\begin{aligned}
& \tilde{z}_{c}(t)=z(t)-\hat{z}^{*}(t), \\
& \tilde{z}_{o}(t)=\hat{z}^{*}(t)-z^{*}(t),
\end{aligned}
$$


converge to zero, since $\tilde{z}(t)=\tilde{z}_{c}(t)+\tilde{z}_{o}(t)$.

\section{Convergence of $\tilde{z}_{o}(t)$}

The signal $\tilde{z}_{o}(t)$ can be seen as the observation error on the reference trajectory. It is directly related to the observation error since :

$$
\left\|\tilde{z}_{o}(t)\right\|=\left\|\kappa\left(v_{c}^{*}, \operatorname{sat}_{1}\left(\hat{v}_{\varepsilon}^{*}(t)\right), \operatorname{sat}_{2}\left(i_{\varepsilon}^{*}(t)\right)\right)-\kappa\left(v_{c}^{*}, v_{\varepsilon}^{*}(t), i_{\varepsilon}^{*}(t)\right)\right\| .
$$

The function $\kappa$ is Lipschitz in its arguments over the domain of interest, then one can use the mean value theorem which states that there exists $L_{1}>0$ such that

$$
\left\|\tilde{z}_{o}(t)\right\| \leq L_{1}\left(\left\|\operatorname{sat}_{1}\left(\hat{v}_{\varepsilon}^{*}(t)\right)-v_{\varepsilon}^{*}(t)\right\|+\left\|\operatorname{sat}_{2}\left(\hat{i}_{\varepsilon}^{*}(t)\right)-i_{\varepsilon}^{*}(t)\right\|\right)
$$

Since $v_{\varepsilon}^{*}(t)$ and $i_{\varepsilon}^{*}(t)$ belong to the unsaturated domain and the sat () function is 1-Lipschitz, equation (43) becomes

$$
\left\|\tilde{z}_{o}(t)\right\| \leq L_{1}\left(\left\|\hat{v}_{\varepsilon}^{*}(t)-v_{\varepsilon}^{*}(t)\right\|+\left\|\hat{i}_{\varepsilon}^{*}(t)-i_{\varepsilon}^{*}(t)\right\|\right)
$$

Using the convergence of the observer, one obtain that if $\theta$ is taken sufficiently large, one has

$$
\left\|\tilde{z}_{o}(t)\right\| \leq L_{1} \alpha \theta e^{-\mu_{\theta} t}\left(\left\|\hat{v}_{\varepsilon}^{*}(t)-v_{\varepsilon}^{*}(0)\right\|+\left\|\hat{i}_{\varepsilon}^{*}(t)-i_{\varepsilon}^{*}(0)\right\|\right) .
$$

where $\alpha$ and $\mu_{\theta}$ are given by proposition 2 .

\section{Convergence of $\tilde{z}_{c}(t)$}

According to (40) and (41), the error $\tilde{z}_{c}(t)$ is ruled by the following dynamical system:

$$
\dot{\tilde{z}}_{c}(t)=A_{c} \tilde{z}_{c}(t)+B_{c} \bar{f}(z(t)) \mu(t)+\bar{\varphi}(z(t))-\dot{\hat{z}}^{*}(t)
$$

where $A_{c}, B_{c}, \bar{f}$ and $\bar{\varphi}$ are the same as in the proof of proposition 1.

Similarly to the proposition 1 , the control law is taken as:

$$
\mu(t)=-\frac{1}{\bar{f}_{e}\left(z(t) ; \hat{v}_{\varepsilon}, \hat{i}_{\varepsilon}\right)} k_{c} B_{c}^{T} \bar{S} \Gamma_{\lambda}\left(\hat{z}(t)-\hat{z}^{*}(t)\right),
$$

where $\bar{f}_{e}\left(z ; \hat{v}_{\varepsilon}, \hat{i}_{\varepsilon}\right) \triangleq f_{e}\left(\psi^{-1}\left(v_{c}, i_{l}, u ; \hat{v}_{\varepsilon}, \hat{i}_{\varepsilon}\right) ; \hat{v}_{\varepsilon}, \hat{i}_{\varepsilon}\right)$, which will be simply denoted $\bar{f}_{e}(z)$, in the sequel. The matrices $\Gamma_{\lambda}$ and $\bar{S}$ are defined by (32) and (33) respectively and $\hat{z}$ by $\hat{z}=\psi\left(v_{c}, i_{l}, u ; s a t_{1}\left(\hat{v}_{\varepsilon}\right), \operatorname{sat}_{2}\left(\hat{i}_{\varepsilon}\right)\right)$.

Note that the corrective term in the proposed control law is not $\tilde{z}_{c}$, because $z$ is not available, indeed, it depends on $v_{\varepsilon}$ and $i_{\varepsilon}$. Let $\bar{z}_{c}=\Gamma_{\lambda} \tilde{z}_{c}$, then

$$
\dot{\bar{z}}_{c}=\lambda A_{c} \bar{z}_{c}-k_{c} \frac{\bar{f}(z)}{\bar{f}_{e}(z)} \lambda B_{c} B_{c}^{T} \bar{S}\left(\bar{z}_{c}+(\hat{z}-z)\right)+\Gamma_{\lambda}\left(\bar{\varphi}(z)-\dot{z}^{*}\right)
$$

since $\Gamma_{\lambda} A_{c} \Gamma_{\lambda}^{-1}=\lambda A_{c}$ and $\Gamma_{\lambda} B_{c}=\lambda B_{c}$.

Let us denote $V$ the following candidate Lyapunov function:

$$
V\left(\bar{z}_{c}\right)=\bar{z}_{c}^{T} \bar{S}_{\bar{c}}
$$

its derivative is given by :

$$
\dot{V}\left(\bar{z}_{c}\right)=2 \lambda \bar{z}_{c}^{T} \bar{S} A_{c} \bar{z}_{c}+2 \bar{z}_{c}^{T} \bar{S} \Gamma_{\lambda} \bar{\varphi}(z)-2 k_{c} \frac{\bar{f}(z)}{\bar{f}_{e}(z)} \lambda \bar{z}_{c}^{T} \bar{S} B_{c} B_{c}^{T} \bar{S}_{\bar{c}}
$$

$$
-2 \bar{z} c \bar{S} \Gamma_{\lambda} \dot{z}^{*}-2 k_{c} \frac{\bar{f}(z)}{f_{e}(z)} \lambda \bar{z}_{c}^{T} \bar{S} B_{c} B_{c}^{T} \bar{S} \Gamma_{\lambda}(\hat{z}-z)
$$


Denoting $V_{1}=2 \lambda \bar{z}_{c}^{T} \bar{S} A_{c} \bar{z}_{c}-2 k_{c} \frac{\bar{f}(z)}{\bar{f}_{e}(z)} \lambda \bar{z}_{c}^{T} \bar{S} B_{c} B_{c}^{T} \bar{S} \bar{z}_{c}$, using the expression of $\bar{S} A_{c}$ deduced from the algebraic Lyapunov equation (33) and taking $k_{c}$ such that

$$
k_{c}>\frac{1}{2}\left(\frac{f_{\max }}{f_{\min }}\right)
$$

leads to

$$
V_{1} \leq-\lambda V\left(\bar{z}_{c}\right)
$$

where $f_{\max }, f_{\min }>0$, are such that $f_{\min } \leq f\left(v_{c}, i_{l}, u ; v_{\varepsilon}, i_{\varepsilon}\right) \leq f_{\max }$, these bounds exist since $\left(v_{c}, i_{l}, u, v_{\varepsilon}, i_{\varepsilon}\right)$ belong to a bounded set given by (2).

Noticing that $\varphi\left(z^{*}\right)=0$ when $z^{*}$ is constant, and denoting $V_{2}=2 \bar{z}_{c}^{T} \bar{S} \Gamma_{\lambda} \bar{\varphi}(z)-2 k_{c} \frac{\bar{f}(z)}{f_{e}(z)} \lambda \bar{z}_{c}^{T} \bar{S} B_{c} B_{c}^{T} \bar{S} \Gamma_{\lambda}(\hat{z}-z)$, gives

$$
V_{2}=-2 k_{c} \frac{\bar{f}(z)}{\bar{f}_{e}(z)} \lambda \bar{z}_{c}^{T} \bar{S} B_{c} B_{c}^{T} \bar{S} \Gamma_{\lambda}(\hat{z}-z)
$$

$$
+2 \bar{z}_{c}^{T} \bar{S} \Gamma_{\lambda}\left(\left(\bar{\varphi}(z)-\bar{\varphi}\left(\hat{z}^{*}\right)\right)+\left(\bar{\varphi}\left(\hat{z}^{*}\right)-\bar{\varphi}\left(z^{*}\right)\right)\right)
$$

Using the Cauchy-Schwartz inequality and applying the mean value theorem, since $\bar{\varphi}$ has a triangular structure and is Lipschitz over its definition range, gives the existence of $c_{1}, c_{2}, c_{3}>0$ such that

$$
V_{2} \leq c_{1} V\left(\bar{z}_{c}\right)+c_{2} \sqrt{V\left(\bar{z}_{c}\right)}\left\|\Gamma_{\lambda} \tilde{z}_{o}\right\|+c_{3} \sqrt{V\left(\bar{z}_{c}\right)}\|\hat{z}-z\|
$$

where $c_{1}$ and $c_{2}$ does not depend on $\lambda$.

Denoting $W(t)=\sqrt{V\left(\bar{z}_{c}(t)\right)}$, using inequalities (50), (52), (53) and Cauchy-Schwartz inequality for the term $-2 \bar{z}_{c}^{T} \bar{S} \Gamma_{\lambda} \hat{z}^{*}$ yields

$$
\dot{W}(t) \leq-\frac{\lambda-c_{1}}{2} W(t)+\frac{c_{2}}{2}\left\|\Gamma_{\lambda} \dot{z}^{*}\right\|+\frac{c_{2}}{2}\left\|\Gamma_{\lambda} \tilde{z}_{o}\right\|+\frac{c_{3}}{2}\|\hat{z}-z\|
$$

It has already been proven that $\tilde{z}_{o}(t)$ converges exponentially toward zero, the same can be done for $\dot{\hat{z}}^{*}(t)$ and $(\hat{z}-z)$ by using the fact that

$$
\begin{aligned}
\dot{\hat{z}}^{*}(t) & =\dot{\tilde{z}}_{o}(t), \\
\|\hat{z}-z\| & =\| \psi\left(v_{c}, i_{l}, u ; \operatorname{sat}_{1}\left(\hat{v}_{\varepsilon}\right), \operatorname{sat}_{2}\left(\hat{i}_{\varepsilon}\right)\right) \\
& \quad-\psi\left(v_{c}, i_{l}, u ; v_{\varepsilon}, i_{\varepsilon}\right) \|, \\
\leq & L_{3}\left(\left\|v_{\varepsilon}-\hat{v}_{\varepsilon}\right\|+\left\|i_{\varepsilon}-\hat{i}_{\varepsilon}\right\|\right),
\end{aligned}
$$

where $L_{3}>0$ exists since $\psi$ is Lipschitz over its domain of definition. Thus, there exist $\alpha, \beta>0$ such that

$$
\dot{W}(t) \leq-\frac{\left(\lambda-c_{1}\right)}{2} W(t)+\alpha e^{-\beta t}
$$

Note that $\beta$ does not depend on $\lambda$ but $\alpha$ may depend on $\lambda$. Furthermore, using the comparison lemma 3.4 in [53], one obtains :

$$
\begin{aligned}
W(t) & \leq W(0) e^{-\frac{\left(\lambda-c_{1}\right)}{2} t}+W(0) \int_{0}^{t} e^{-\frac{\left(\lambda+c_{1}\right)}{2}(t-s)} \alpha e^{-\beta s} d s \\
& \leq W(0) e^{-\frac{\left(\lambda-c_{1}\right)}{2} t}+\alpha W(0) e^{-\frac{\left(\lambda+c_{1}\right)}{2} t}\left[\frac{e^{-\left(\beta-\frac{\lambda-c_{1}}{2}\right) s}}{-\left(\beta-\frac{\lambda-c_{1}}{2}\right)}\right]_{0}^{t} \\
& \leq W(0) e^{-\frac{\left(\lambda-c_{1}\right)}{2} t}+\frac{\alpha W(0) e^{-\frac{\left(\lambda+c_{1}\right)}{2} t}}{\frac{\lambda-c_{1}}{2}-\beta}\left(e^{\left(\frac{\lambda-c_{1}}{2}-\beta\right) t}-1\right) \\
& \leq W(0) e^{-\frac{\left(\lambda-c_{1}\right)}{2} t}+\frac{\alpha W(0)}{\frac{\lambda-c_{1}}{2}-\beta}\left(e^{-\beta t}-e^{-\frac{\lambda-c_{1}}{2} t}\right)
\end{aligned}
$$


Finally, if $\lambda$ is chosen so that

$$
\lambda>c_{1}
$$

then equation (56) implies the exponential convergence of $W$ toward zero and thus of $V$. If $\lambda$ is taken sufficiently large

$$
\left\|\tilde{z}_{c}(t)\right\| \leq \alpha_{\lambda}^{c} e^{-\min \left\{\left(\lambda-c_{1}\right), \beta\right\} t}
$$

where $\alpha_{\lambda}^{c}>0$. This ends the proof.

\section{References}

[1] Sahu, B., and Rincón-Mora, G., 2004. "A low voltage, dynamic, noninverting, synchronous buck-boost converter for portable applications". IEEE Transactions on Power Electronics, 19(2), pp. 443-452.

[2] Walker, G., and Sernia, P., 2004. "Cascaded dc-dc converter connection of photovoltaic modules". IEEE Transactions on Power Electronics, 19(4), pp. 1130-1139.

[3] Khaligh, A., Rahimi, A., and Emadi, A., 2008. "Modified pulse-adjustment technique to control dc/dc converters driving variable constant-power loads”. IEEE Transactions on Industrial Electronics, 55(3), pp. 1133-1146.

[4] Jin, K., Ruan, X., Yang, M., and Xu, M., 2009. “A hybrid fuel cell power system”. IEEE Transactions on Industrial Electronics, 56(4), pp. 1212-1222.

[5] Erickson, R., and Maksimovic, D., 2001. Fundamentals of power electronics, 2 ed. Springer-Verlag, New-York.

[6] Redl, R., and Sokal, N., 1986. "Near-optimum dynamic regulation of dc-dc converters using feed-forward of output current and input voltage with current-mode control". IEEE Transactions on Power Electronics, 3(3), pp. 181-192.

[7] Ridley, R., 1991. "A new, continuous-time model for current-mode control”. IEEE Transactions on Power Electronics, 6(2), pp. 271-280.

[8] Maksimovic, D., Zane, R., and Erickson, R., 2004. "Impact of digital control in power electronics". In International Symposium on Power Semiconductor Devices ICs, IEEE, pp. 13-22.

[9] Morales, J., Leyva-Ramos, J., Carbajal, E., and Ortiz-Lopez, M., 2008. "Average current-mode control scheme for a quadratic buck converter with a single switch". IEEE Transactions on Power Electronics, 23(1), pp. $485-490$.

[10] Olalla, C., Leyva, R., El Aroudi, A., and Garces, P., 2009. "Qft robust control of current-mode converters: application to power conditioning regulators". International Journal of Electronics, 96(5), pp. 503-520.

[11] Chang, Y., and Lai, Y., 2009. "Parameter tuning method for digital power converter with predictive current-mode control”. IEEE Transactions on Power Electronics, 24(12), pp. 2910-2919.

[12] Leyva-Ramos, J., and Morales-Saldana, J., 2000. "Uncertainty models for switch-mode dc-dc converters". IEEE Transactions on Circuits and Systems I: Fundamental Theory and Applications, 47(2), pp. 200-203.

[13] Leung, F., Tam, P., and Li, C., 1991. "The control of switching dc-dc converters-a general lwr problem". IEEE Transactions on Industrial Electronics, 38(1), pp. 65-71.

[14] Leung, F., Tam, P., and Li, C., 1993. "An improved lqr-based controller for switching dc-dc converters". IEEE Transactions on Industrial Electronics, 40(5), pp. 521-528.

[15] Garofalo, F., Marino, P., Scala, S., and Vasca, F., 1994. "Control of dc-dc converters with linear optimal feedback and nonlinear feedforward". Power Electronics, IEEE Transactions on, 9(6), pp. 607-615.

[16] Leyva, R., Martínez-Salamero, L., Valderrama-Blavi, H., Maixé, J., Giral, R., and Guinjoan, F., 2001. "Linear statefeedback control of a boost converter for large-signal stability”. IEEE Transactions on Circuits and Systems I: Fundamental Theory and Applications, 48(4), pp. 418-424.

[17] Olalla, C., Leyva, R., El Aroudi, A., and Queinnec, I., 2009. "Robust lqr control for pwm converters: an lmi approach". IEEE Transactions on Industrial Electronics, 56(7), pp. 2548-2558.

[18] Olalla, C., Leyva, R., El Aroudi, A., Garces, P., and Queinnec, I., 2010. "Lmi robust control design for boost pwm converters". IET Power Electronics, 3(1), pp. 75-85.

[19] Olalla, C., Queinnec, I., Leyva, R., and El Aroudi, A., 2012. “Optimal state-feedback control of bilinear dc-dc converters with guaranteed regions of stability”. IEEE Transactions on Industrial Electronics, 59(10), pp. 3868-3880.

[20] Olalla, C., Leyva, R., Queinnec, I., and Maksimovic, D., 2012. "Robust gain-scheduled control of switched-mode dc-dc converters". IEEE Transactions on Power Electronics, 27(6), pp. 3006-3019.

[21] Shirazi, M., Zane, R., and Maksimovic, D., 2009. "An autotuning digital controller for dc-dc power converters based on online frequency-response measurement". IEEE Transactions on Power Electronics, 24(11), pp. 2578-2588.

[22] Ebrahimzadeh, M., and Rahmati, A., 2010. "Adaptive and fast-response controller for boost pfc converter with wide range of operating conditions". In Power Electronic \& Drive Systems \& Technologies Conference (PEDSTC), 2010 1st, IEEE, pp. 157-162. 
[23] Sira-Ramirez, H., 1991. "Nonlinear pi controller design for switchmode dc-to-dc power converters". IEEE Transactions on Circuits and Systems, 38(4), pp. 410-417.

[24] Beccuti, A., Mariethoz, S., Cliquennois, S., Wang, S., and Morari, M., 2009. "Explicit model predictive control of dc-dc switched-mode power supplies with extended kalman filtering". IEEE Transactions on Industrial Electronics, 56(6), pp. 1864-1874.

[25] Sira-Ramírez, H., Ortega, R., and García-Esteban, M., 1998. "Adaptive passivity-based control of average dc-to-dc power converter models". International journal of adaptive control and signal processing, 12(1), pp. 63-80.

[26] Leyva, R., Cid-Pastor, A., Alonso, C., Queinnec, I., Tarbouriech, S., and Martinez-Salamero, L., 2006. "Passivity-based integral control of a boost converter for large-signal stability”. IEE Proceedings-Control Theory and Applications, 153(2), pp. 139-146.

[27] Sanders, S., and Verghese, G., 1992. "Lyapunov-based control for switched power converters". IEEE Transactions on Power Electronics, 7(1), pp. 17-24.

[28] Alonge, F., D'Ippolito, F., Raimondi, F., and Tumminaro, S., 2007. "Nonlinear modeling of dc/dc converters using the hammerstein's approach”. IEEE Transactions on Power Electronics, 22(4), pp. 1210-1221.

[29] Alonge, F., D'Ippolito, F., and Cangemi, T., 2008. "Identification and robust control of dc/dc converter hammerstein model”. IEEE Transactions on Power Electronics, 23(6), pp. 2990-3003.

[30] Feng, G., Meyerc, E., and Liu, Y., 2007. "A new digital control algorithm to achieve optimal dynamic performance in dc-to-dc converters". IEEE Transactions on Power Electronics, 22(4), pp. 1489-1498.

[31] Nazarzadeh, J., and Jafarian, M., 2013. "Applying bilinear time-optimal control system in boost converters". IET Power Electronics, 7(4), pp. 850-860.

[32] El Fadil, H., Giri, F., El Magueri, O., and Chaoui, F., 2009. "Control of dc-dc power converters in the presence of coil magnetic saturation”. Control Engineering Practice, 17(7), pp. 849-862.

[33] Mattavelli, P., Rossetto, L., Spiazzi, G., and Tenti, P., 1995. "General-purpose fuzzy controller for dc/dc converters". In Applied Power Electronics Conference and Exposition, IEEE, pp. 723-730.

[34] Perry, A., Feng, G., Liu, Y., and Sen, P., 2007. "A design method for pi-like fuzzy logic controllers for dc-dc converter". Industrial Electronics, IEEE Transactions on, 54(5), pp. 2688-2696.

[35] Wai, R., 2001. "Total sliding-mode controller for pm synchronous servo motor drive using recurrent fuzzy neural network". IEEE Transactions on Industrial Electronics, 48(5), pp. 926-944.

[36] Wai, R., and Shih, L., 2012. "Adaptive fuzzy-neural-network design for voltage tracking control of a dc-dc boost converter". IEEE Transactions on Power Electronics, 27(4), pp. 2104-2115.

[37] Oucheriah, S., and Guo, L., 2013. "Pwm-based adaptive sliding-mode control for boost dc-dc converters". IEEE Transactions on Industrial Electronics, 60(8), pp. 3291-3294.

[38] Sira-Ramirez, H., 1987. "Sliding motions in bilinear switched networks". IEEE Transactions on Circuits and Systems, 34(8), pp. 919-933.

[39] Tan, S., Lai, Y., Tse, C., Martínez-Salamero, L., and Wu, C., 2007. "A fast-response sliding-mode controller for boost-type converters with a wide range of operating conditions". IEEE Transactions on Industrial Electronics, 54(6), pp. 3276-3286.

[40] Tan, S., Lai, Y., and Tse, C., 2008. "General design issues of sliding-mode controllers in dc-dc converters". IEEE Transactions on Industrial Electronics, 55(3), pp. 1160-1174.

[41] Wai, R., and Shih, L., 2011. "Design of voltage tracking control for dc-dc boost converter via total sliding-mode technique". IEEE Transactions on Industrial Electronics, 58(6), pp. 2502-2511.

[42] Bédoui, A., Farza, M., MSaad, M., and Ksouri, M., 2008. "Robust nonlinear controllers for bioprocesses". In Proc. of the IFAC World Congress, Seoul, pp. 15541-15546.

[43] O’Reilly, J., 1983. Observers for linear systems, Vol. 170. Academic Press.

[44] Astrom, K., and Hagglund, T., 2006. Advanced PID control. ISA, Advanced PID control.

[45] Oukaour, A., Pouliquen, M., Tala-Ighi, B., Gualous, H., Pigeon, E., Gehan, O., and Boudar, B., 2013. "Supercapacitors aging diagnosis using least square algorithm". Microelectronics Reliability, 53, pp. 1638-1642.

[46] Farza, M., MSaad, M., and Rossignol, L., 2004. "Observer design for a class of mimo nonlinear systems". Automatica, 40(1), pp. 135-143.

[47] Khalil, H., and Esfandiari, F., 1993. "Semiglobal stabilization of a class of nonlinear systems using output feedback". IEEE Transactions on Automatic Control, 38(9), pp. 1412-1415.

[48] European Cooperation for Space Standardization, 2004. ECSS : Electrical and Electronic Standart : ECSS-E20A.

[49] Farza, M., Othman, S., Hammouri, H., and Fick, M., 1997. "Discrete-time nonlinear observer-based estimators for the on-line estimation of the kinetic rates in bioreactors". Bioprocess Engineering, 17(4), pp. 247-255.

[50] Farza, M., Busawon, K., and Hammouri, H., 1998. "Simple nonlinear observers for on-line estimation of kinetic rates in bioreactors". Automatica, 34(3), pp. 301-318.

[51] Farza, M., M’Saad, M., Fall, L., Pigeon, E., Gehan, O., and Mosrati, R., 2014. “Continuous-Discrete Time Observer for a class of MIMO Nonlinear Systems”. IEEE Transactions on Automatic Control, Apr., pp. 1060-1065. 
[52] Gauthier, J., Hammouri, H., and Othman, S., 1992. "A simple observer for nonlinear systems applications to bioreactors". IEEE Transactions on Automatic Control, 37(6), pp. 875-880.

[53] Khalil, H., 2002. Nonlinear Systems. Prentice Hall PTR. 